\title{
Do Healthcare Metadata Models Designed for Web Publishing Meet the Accreditation Standards? A Case Study in the Healthcare and Medical Education
}

\author{
Reem Q. Al Fayez \\ Department of Computer Information Systems, King Abdullah || School for Information \\ Technology, Department of Computer Information Systems, The University of Jordan, Amman, \\ Jordan \\ r.alfayez@ju.edu.jo \\ DOI: 10.34190/EJEL.20.18.4.007
}

\begin{abstract}
This article reports comparison results from studying the alignment of selected metadata models, used to manage E-Learning Materials (ELM) in the medicine and healthcare education, against the accreditation standards of the European Accreditation Council for Continuing Medical Education (EACCME). The study aims to (1) illustrate the variety of application profiles developed as metadata models in that field, (2) identify the alignment of the metadata models with the criteria set by the EACCME. This study compared several application profiles, developed based on established metadata standards, using an evaluation matrix created using the accreditation criteria set by the EACCME. Further technical criteria were added to compare between the metadata models and their alignment with the new techniques used for publishing data online. Such techniques aid in the searchabililty and discovery of online content via the internet. Therefore, the use of XML and RDF technologies combined with the use of ontologies and controlled vocabulary was emphasized in the implementation of many metadata models. One limitation faced during conducting this research was the poor documentation of the methodology for designing the metadata models. Despite this, it is apparent that the EACCME criteria can work as best practices guidelines for describing the content of digital libraries with medical and healthcare content in order to be accredited later on. The study showed that the medicine and healthcare educational content requires specialized metadata models that consider having specific properties such as clinical history and expiry time of its material shown in some of the application profiles. Further research should be conducted to evaluate the completeness and conformity of applying the application profiles in digital libraries and online repositories to the standards' guidelines.
\end{abstract}

Keywords: Health Educators; Medical Education; Accreditation; Library Materials; Electronic E-learning Materials; Quality of Education

\section{Introduction}

Medicine and healthcare disciplines are considered one of the early adopters for technology advances related to learning (Reid, Thomson and McGlade, 2016). The online educational content provided for the medical and healthcare professions education is now used to effectively support the learning process (Fleiszer, Posel and Steacy, 2004). Materials provided on the internet are considered a source that has the potential for improving the new learning paradigms such as blended learning and continuing professional development (CPD) in medical education (Delf, 2013). Several e-platforms and e-libraries are providing high-quality learning content for the public (Caswell et al., 2008). Such platforms might be under the supervision of universities or medical centers but that is not always the case. Understanding the importance of e-learning, the European Accreditation Council for Continuing Medical Education (EACCME) set up by the European Union of Medical Specialists (UEMS) has launched in 2009 the accreditation of e-learning materials (ELM) and that includes recordings, videos, and any online material published online via an educational website (UEMS, 2016a).

There exist standards agreed upon for publishing data online. A standard, in general, is "a document, established by a consensus of subject matter experts and approved by a recognized body that provides guidance on the design, use or performance of materials, products, processes, services, systems or persons" (ISO, 2019). In the context of e-learning materials, many standards were developed to govern the description, design, and publishing of e-learning materials for ensuring reusability, and interoperability between different e-learning systems (Koutsomitropoulos et al., 2010). However, it is possible that a standard does not satisfy specific systems' or publishers' needs. Hence, Application Profiles (AP) can be developed to implement a modified version of any standard, either by adding or removing characteristics from the standard's set of attributes and still guarantee compatibility with the original standard, and interoperability of its content (Issam, Passardiere and Jean-marc, 2008). 
As several specialized application profiles have been developed for describing medical and healthcare educational content, this emphasized the existence of specific requirements when describing such content. Some of these requirements are the use of specific controlled vocabularies known in the medical domain for enhancing the discoverability and the organization of its content. Furthermore, properties such as clinical history, patient information, target audience, and continuing education credit are specialized properties for describing medical and healthcare content in specific. Specialized healthcare organizations have been developing metadata standards that are used by the healthcare community to advance healthcare education (Hersh et al., 2006). The usage of the metadata standards depends on the organization adopting it. Online libraries and educational repositories have different goals when publishing its content online. Some might have the goal to enhance the searchability and discoverability of its content (Konstantinidis, Kaldoudi and Bamidis, 2009), while other online libraries contain types of e-learning materials that is of different media types such as videos and interactive lessons, have technical details to be included in its description.

Furthermore, it is important that online libraries are up to date with digitizing movement happening in the world. Medical and healthcare data are increasingly published as open data on the web. Hence, ontologies in general and biomedical ontologies in specific are used heavily to aid in the searchability and integration of such data (Issam, Passardiere and Jean-marc, 2008). Ontologies are controlled vocabularies managed by experts in their fields that allow describing the semantics of data in machine readable way (Konopka, 2015). RDF is one of the standards used to represent the ontologies. It provides the foundation for describing resources on the web in semantically enriched manner and enables data interoperability (Ashraf, Hussain and Hussain, 2014). Several biomedical and more general ontologies are used for describing medical educational content in online libraries. For example, Medical Subject Headings (MeSH) ontology which is a controlled vocabulary used by PubMed library for indexing articles (Dhammi and Kumar 2014), Systematized Nomenclature of Medicine - Clinical Terms (SNOMED CT) used heavily to effectively record clinical data and it covers symptoms, procedures, diagnosis, and other important medical terms (Stearns et al., 2001), and Unified Medical Language System (UMLS) that brings health and biomedical vocabularies and coding standards to enable interoperability between medical information systems (Bodenreider, 2004). Such ontologies are specialized in describing medical terminologies and used heavily by medical and healthcare organizations. While, other more general ontologies such as Simple Knowledge Organization System (SKOS) that is used to represent knowledge using RDF (Miles et al., 2005), and Friend of a Friend (FAOF) that is used for linking information and using the web (Graves, Constabaris and Brickley, 2007).

This paper is organized as follows. First, a summary of existing application profiles developed for the healthcare e-learning materials in specific is provided in the background section. The summary highlights the elements of each application profile and outlines their structure graphically. Next, the paper outlines the methodology and criteria used to conduct a comparative study for the application profiles against the accreditation criteria set by the EACCME for accrediting ELMs in terms of its description. Furthermore, the study added criteria to compare the technical implementation techniques used by these application profiles of metadata models to enhance the description of ELM in medicine and healthcare. At the end of this study, discussion and conclusion are presented to compares the alignment of the metadata models with the accreditation standards.

\section{Background}

Two of the most well-established metadata standards are described in this section. Understanding the elements of such standards will ease the understanding of the application profiles compared in this study.

\subsection{The DCMI}

Dublin Core Metadata Initiative (DCMI) is an open public organization that is non-profitable. It supports metadata design and implementation to support resource discovery around the internet.. The initiative work resulted in a simple cross-domain metadata statement known as Dublin Core Metadata Element Set (DCMES) which has been standardized and revised as ISO standard 15836-1: 2017 (ISO, 2017). Dublin Core standard is used to describe a wide range of resources, where a resource is defined by DCMI as "anything which might be identified" (Powell et al., 2007) . DCMI used the same definition of RDF for a resource. A resource is "anything that has identity" (Maicher, 2007). Therefore, in online libraries, any book, book chapter, article, or any multimedia object can be described is referred to as a resource. The simple Dublin Core metadata element set consists of these fifteen elements: contributor, coverage, creator, date, description, format, identifier, language, 
publisher, relation, rights, source, subject, title, and type. All these elements are optional and may be repeated if required for a single resource.

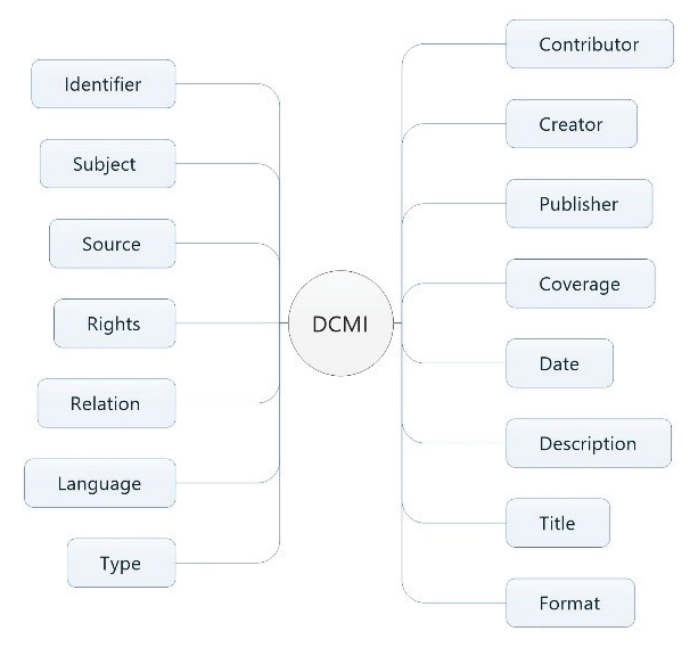

Figure 1: DCMI metadata standard

The work on DCMES started in 1998. Since then, the field of metadata standards has developed (Barker and Campbell, 2010). The DCMI continued its work on developing these simple elements set to accommodate the needs of semantic web and interoperability of a resource. In 2008, DCMI published a set of specifications to conform to the new trends in the field such as using RDF (W3C, 2014) to represent metadata fields. DCMI metadata terms are the new version of DCMI simple element set. The new metadata terms are 15 new properties identical to the old 15 elements in the naming. Hence, the interoperability of existing implementations of the Simple Element Set is not be affected. The new 15 elements in DCMI metadata terms have been assigned domains and ranges and are related with the previous DCMES as sub-properties through RDF tags. Domains and ranges restrict the content of a given property to specific types and values. The main elements of the standards are illustrated in figure 1.

The element set of DCMI describe the basic characteristics of a resource and its relations with other resources such as persons using creator and contributor properties with the aid of ontologies. Classes are part of vocabulary model in any metadata standard or application profile (Maicher, 2007). Syntax and vocabulary encoding schemas are used to describe the type and language of a resource from a selection of options provided by the standard.

\subsection{The IEEE LOM}

IEEE Learning Technology Standards Committee (LTSC) started to develop the Learning Object Metadata (LOM) standard in 1997. With the aid of different international participants, LOM working group succeeded to have this standard accredited by IEEE. In June 2002, the 1484.12.1 LOM standard was accredited and published by IEEE (IEEE Computer Society, 2002) and the IEEE LTSC LOM group continued to develop and maintain the standard ever since. As defined in LOM standard (IEEE Computer Society, 2002), a LOM instance is designed to record the characteristics of the learning object it describes. LOM categorizes these characteristics into 9 groups: general, life cycle, meta-metadata, educational, technical, rights, relation, annotation, and classification. In each of these categories, there are a set of data elements which compose a metadata instance for a learning object. The purpose of such a detailed description for a learning object, as stated by the working group, is to facilitate sharing and exchange of learning objects since the metadata have a high degree of semantic interoperability. The total number of elements composing LOM standard is 45 elements which are all optional to complete in this standard. These 45 elements are directly descendent from the 9 parent categories and each element might be a simple data element (leaf node) or an aggregate element for collection of simple data elements (parent node) as illustrated in figure 2 . 


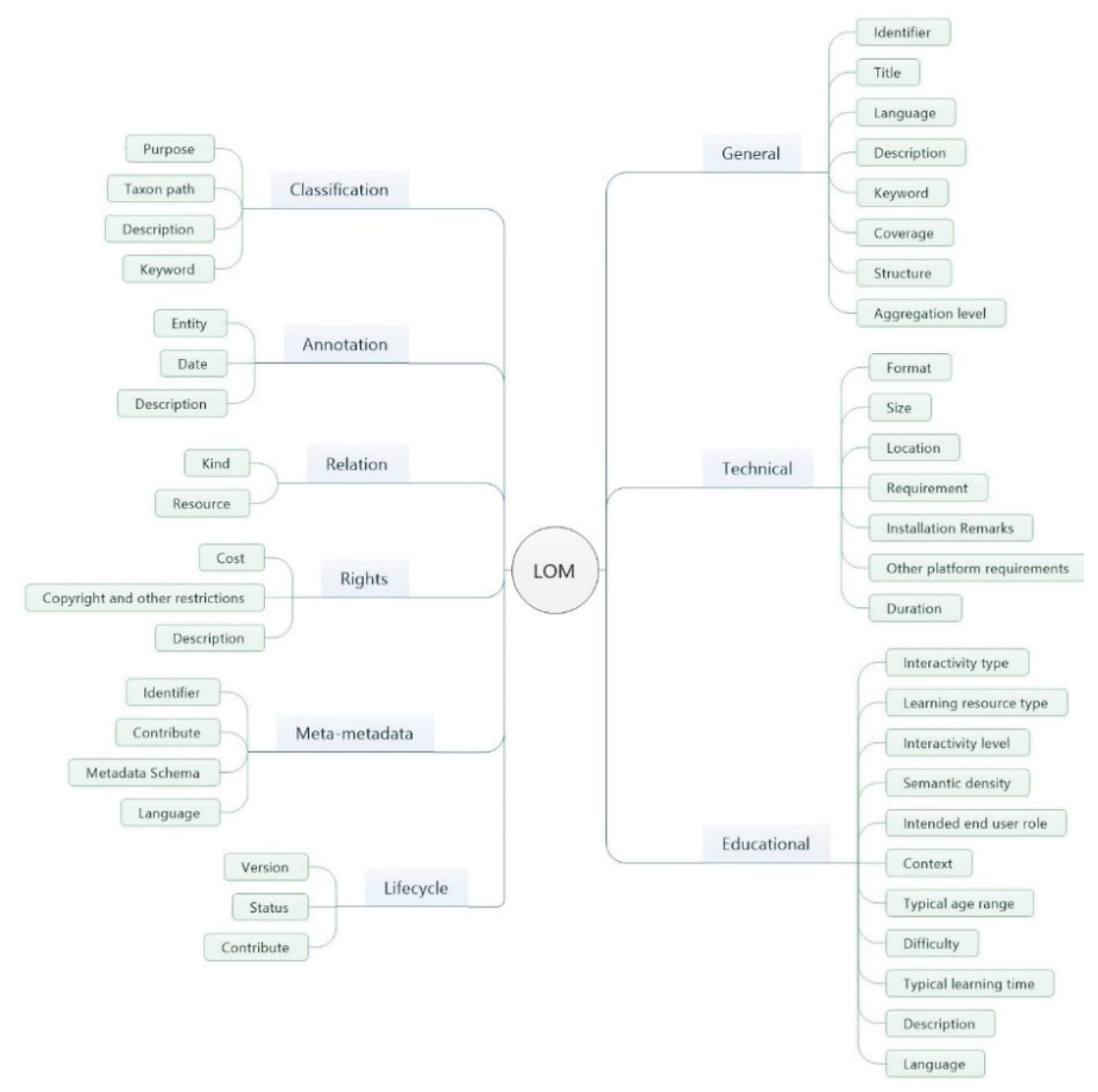

Figure 2: LOM metadat standard

Simple data elements in LOM hierarchy have individual values defined by the standard restrictions on data types and size of the content of these elements. For example, for a simple data element, LOM standard may specify the size of allowed values in the form of the smallest permitted maximum value. Such restriction forces the application implementing this standard to bound the maximum size for a specific element to the smallest permitted value for that element. Another restriction imposed by LOM standards is the type of content in a metadata element. Data types vary in LOM such as DateTime and; LangString, where one part of the value is a language code and the other part is Unicode text; or it might be a vocabulary, where the value of the element is selected from a controlled vocabulary; or character strings; and some elements have undefined data type.

In LOM, some data elements may be repeated in different categories. The value and meaning of an element are affected by the parent category which contains that element, for example, keyword element in the general category contains values that describe the learning object itself, while the keyword element in the classification category contains various fields that this learning object might fit in.

\section{Methodology}

An evaluation matrix is constructed to compare the alignment of different metadata models to the essential criteria set by the EACCME to guarantee the high-level quality of ELMs in medicine and healthcare fields. The criteria focus on content and description quality of the ELMs. The quality of the content cannot be assessed unless evaluated by humans. This section details all the criteria set by the EACCME. The comparison study considers only the part of the criteria related to the description of the ELMs to create the evaluation matrix. Furthermore, the implementation techniques that aligns with the new publishing standards of using RDF and ontologies were added to the evaluation matrix. This study provides a mechanism to compare and evaluate the features presence or absence in each application profiles used as metadata models in the field. Details about the evaluation matrix criteria and scope of the study are detailed below. 


\subsection{Requirements for accreditation of ELMs set by the EACCME}

The reliability on continuing medical education and the greater support for such change in learning has resulted in considerable advances in the practices applied by learners to engage in educational opportunities provided in terms of e-learning material that include e-books, articles, videos, pics, ..., etc. Therefore, the European Union of Medical Specialists (UEMS) build The European Accreditation Council for Continuing Medical Education (EACCME) (UEMS, 2016b). The e-learning materials were defined by the EACCME as materials of new media such as recorded audio, recorded visual on CD or DVD, or available online via an educational website, and not limited to these examples (EACCME, 2017). EACCME required an ELM to satisfy a set of the following essential criteria to gain the accreditation (UEMS, 2016a):

1. Educational objectives and fulfillment of learning needs: which include set of criteria that mandate the provider of the material to state the educational needs which the material fulfill, the educational outcomes of the material, and the target audience for whom the material will be suitable for.

2. Description of material: which include set of criteria that govern the description of the material, by providing summary of its content, the duration the learner needs to engage with the material, its compliance with ethical and legal requirements if applicable, the date of preparation of the material and any revisions made or its expiry date if applicable, the format required for use of this material, and if it respect the privacy and confidentiality of the learner, if the material is of an interactive nature.

3. Nature of material: include set of criteria that assess the content of the material if it complies with the following: it must be evidence-based with suitable references, it must encourage active learning, and it must confirm the learner engagement and achievement of the educational objectives using selfassessment methodologies or other assessment methods such as questionnaires.

4. Details of the provider: include set of criteria that govern the description of the provider, such as the description of the provider organization, the names and qualifications of individuals involved in preparing the material, and it must state hot-links to further relevant information, and the source of funding provided for preparing them when applicable.

5. Quality assurance by the provider: which includes a set of criteria mandates the declaration of the provider techniques applied to ensure the quality of the material developed. Such criteria must be followed by the provider; they must provide confirmation that the material has been quality assured, they must provide means for the learner to provide feedback on the material, and they should make technical support related to the material available for the learner.

\subsection{The study evaluation criteria}

The comparative study is accomplished based on an evaluation matrix created for this purpose. The evaluation matrix contains a subset of the EACCME criteria related to assessing the description of ELMs that include: 1. educational objectives and fulfillment of educational needs, 2. description of the material, and 3. details of the provider. Having such elements in an application profile indicate that it is aligned with the criteria needed for the accreditation standards and guarantee good quality for the ELM metadata. The study did not consider two of EACCME criteria that are related to: nature of material, and the quality assurance by the provider. Such criteria are evaluated based on the content provided in the ELM and how the providers of the content are maintaining it is quality. Since such features are not listed in the metadata of ELMs in general, the study considered the criteria that can be covered and managed by the metadata description of ELMs in online libraries and repositories.

Furthermore, the technical implementation of the application profiles in digital libraries aids in making the ELMs searchable and discoverable. Therefore, the comparative study added technical criteria which cover the binding techniques and controlled vocabulary used when implementing the application profiles, and what metadata standards they extended when developed which ease the interoperability and usability of ELM on the web. One more criterion added that focused on the use of online licensing certificates for recording the IRP right for the resources described in these application profiles.

The comparison is conducted by listing all the criteria explained above in the matrix dimensions and answering it with mostly Yes/NO to compare the application profiles. The resulting matrix is represented in a table format that summarizes and compare the results. The application profiles participating in this comparative study are detailed in the next section. 


\subsection{Comparative study coverage}

As part of the study, the following Application Profiles (APs) were found in previous studies researched and outlined in this section. Such APs were developed specifically to manage medicine and healthcare related ELM. Table 1 lists the participating application profiles in the study and a link for their website that or documentation provided by the agency responsible for developing it.

Table 1: Analysis collection overview

\begin{tabular}{l|l}
\hline Name & URL \\
\hline HealthCare LOM & $\underline{\text { http://ns.medbiq.org/lom/extend/v1// }}$ \\
\hline mEducator & http://www.meducator.net/ \\
\hline$H E A L$ & $\underline{\text { https://library.med.utah.edu/heal/ }}$ \\
\hline$N L M$ & $\underline{\text { http://www.nlm.nih.gov/tsd/cataloging/metafilenew.html }}$
\end{tabular}

\section{Results}

The evaluation matrix in table 2 shows the results of evaluating each application profile against the criteria discussed in the methodology section. The table gives a general overview of the characteristics of each of the participating application profile. More detailed results are explained in this section for each participating application profiles in the study.

\subsection{HealthCare LOM}

Healthcare LOM was developed as an application profile within the MedBiquitous Learning Object Working Group that extends the IEEE Learning Object Metadata (LOM) (Smothers, 2015). In this application profile, one category named HealthCare Metadata was added to the original 9 categories composing LOM, in which fields to describe healthcare related metadata were added (figure 3). Results of evaluating the Healthcare LOM against the evaluation matrix criteria are detailed as follows:

1. Educational objectives and fulfillment of learning needs:

The original LOM provides in its educational category details about the resource type, interactivity level, its difficulty, the context where this resource can be used, and the typical age range. Original LOM and HealthCare LOM did not state explicitly the educational needs and outcomes related to the material it describes. However, in HealthCare metadata category, the target audience element was added to LOM.

2. Description of materials:

The general category of HealthCare LOM has a summary description of a resource. In the HealthCare metadata category, the time required for a learner to use the material can be derived from two fields: start date time and end date time. More dates are stored in the life cycle category such as date of creation, and date of any other versioning of the resource. In addition, the expiration date of the resource is added to the healthcare education category. The technical category in the healthcare LOM provides information that describes the format, size, installation remarks, and additional information for other platform requirements. The rights category describes the licensing of the resource but does not state the privacy and confidentiality of the learner.

3. Details of the provider:

The rights category details information about the provider of the resource, along with restrictions on how this resource is used. The contact field is added in the HealthCare Education category for further information about the provider. Additional fields concerning the commercial and financial support of the resource are added to the healthcare LOM.

4. Technical information

HealthCare LOM extends the XML binding of IEEE LOM. The original IEEE LOM has been developed using XML/RDF format, which makes it easy to implement the application profile in the RDF format too. Healthcare LOM introduced using medical terminologies such as MeSH (Dhammi and Kumar, 2014), SNOMED (Stearns et al., 2001), and UMLS (Bodenreider, 2004) to indicate the keywords of a resource. The IPR of a resource is stored in the rights category and the credits element in the healthcare education category. However, HealthCare LOM does not support the use of any online copyright certificate. It is noted that most of the elements in this application profile are optional. 


\section{2 mEducator}

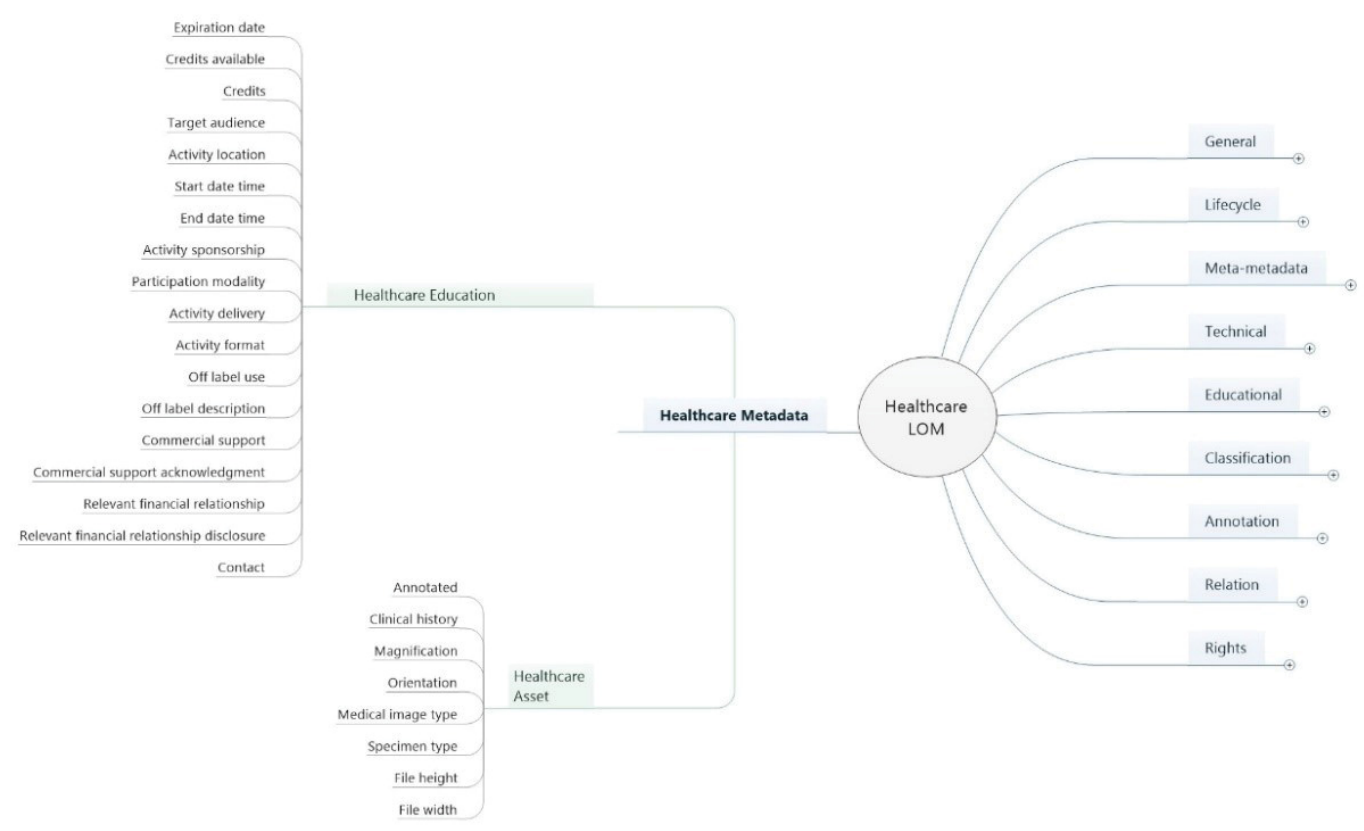

Figure 3: Healthcare LOM application profile

The mEducator project is a European funded best practice network launched to host medical educational materials from European higher academic institutions (Hendrix et al., 2012). It proposed, in 2009, the mEducator application profile (figure 4). The objective of mEducator schema was to enable ease of sharing, discovery, and reuse of medical educational content across EU higher academic institutions. Results of evaluating the mEducator against the evaluation matrix criteria are detailed as follows:

1. Educational objectives and fulfillment of learning needs:

Educational objectives, educational outcomes, and the educational level of a resource are all detailed in the pedagogical values provided by mEducator along with other fields such as learning instructions, assessment methods, and educational pre-requisites.

2. Description of Materials:

Content description, technical description, creation date, keywords, citation, metadata creation date, and others are elements used in the description category of the mEducator schema. Furthermore, information that certifies the content quality are added to the general category, and discipline of the resources are stored in the classification category.

3. Provider details

The basic elements of mEducator include the author of the content and the author of the metadata. Furthermore, the application profile captures repurposing history of the resource it describes using the repurposing category. It has fields to document different information about the parent resource from which the current resource has been created, if it is a repurposed resource

4. Technical information

mEducator extended the Dublin Core Element Set and further elements were proposed to meet mEducator project requirements. The application profile was implemented in both XML and RDF in order to ensure that the metadata was compliant with the principles of Linked Data (Hitzler and Janowicz, 2013). Controlled vocabularies such as FOAF vocabulary can be used to represent people in RDF. Resources types and educational outcomes can be described using SKOS vocabulary. Furthermore, mEducator represents usage rights and respect the rights of content authors using IRP licensing represented in controlled vocabulary based on common creative licensing schema (Creative Commons, 2017). 


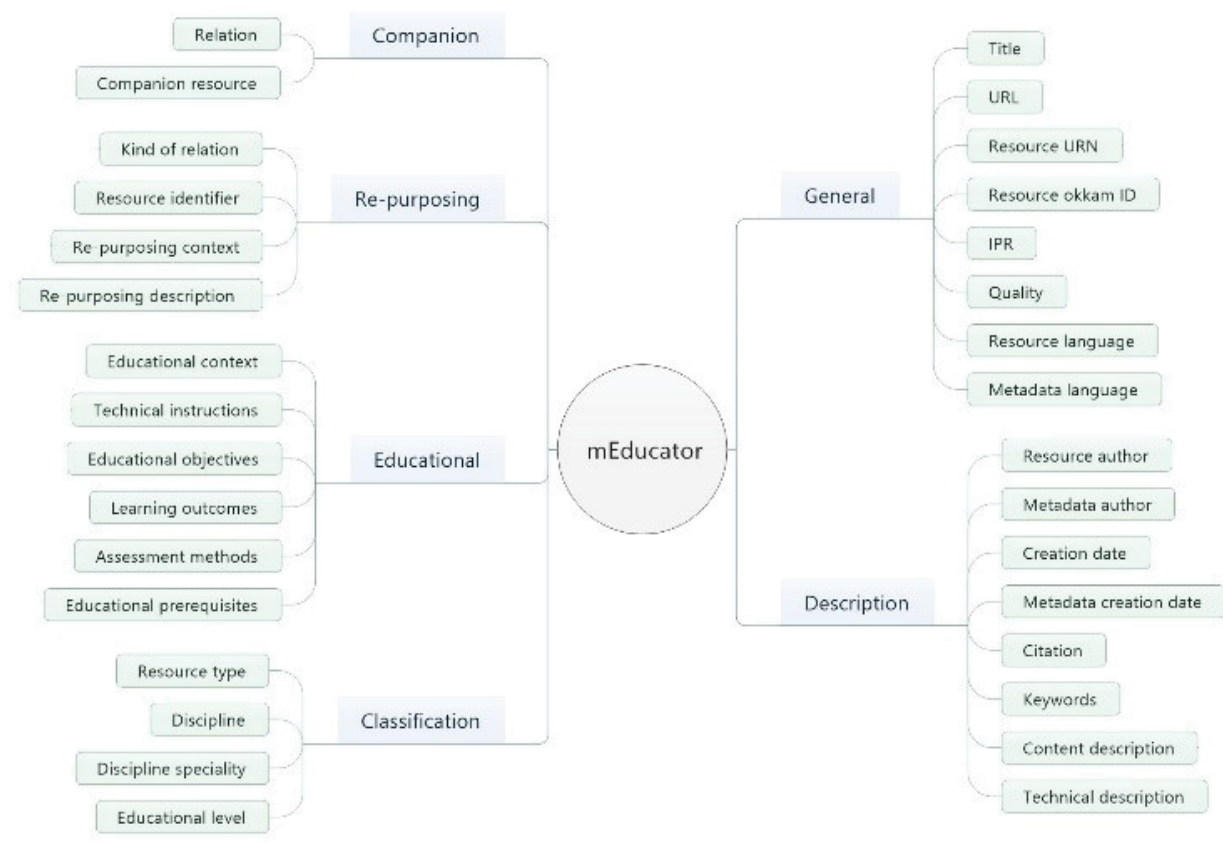

Figure 4: mEducator application profile

\subsection{HEAL}

The Health Education Assets Library (HEAL) is designed to provide health sciences' educators with freely available, high-quality multimedia materials to boost health science education. HEAL project (Candler, Uijtdehaage and Dennis, 2003) is a result of a collaboration between numerous faculties, medical schools, the Association of American Medical Colleges, and the National Library of Medicine. Results of evaluating the HEAL application profile (figure 5) against the evaluation matrix criteria are detailed as follows:

1. Educational objectives and fulfillment of learning needs:

The educational objectives of a resource and its learning needs are not listed in the HEAL application profile. The targeted audience is not explicitly stated, but an element named inappropriate for minor in the extension category.

2. Description of Materials:

HEAL application profile comply with almost all the criteria when describing its material. Summary of the material content is provided in the general category, while the typical learning time is included in the educational category of HEAL and information about software requirements are described in the technical section. Furthermore, the lifecycle category covers the date of preparing the resource and contacts information for the resource creator. The expiry date of the resource is not registered in HEAL. As for the legal and ethical issues, HEAL provides in the rights section clear information about assuring patient confidential content and stating clear intellectual property rights. In terms of learner privacy, HEAL does not clearly state that it respects the learner privacy and confidentiality when interacting with interactive learning resources since it has a special content type and this feature is not applicable in all cases.

3. Provider details:

The contributor to HEAL collection is responsible for providing details about the organizations or individuals involved in preparing the resource uploaded in the life cycle category. One of the elements from HEAL extensions provide URLs pointing to educational context in hich the resource can be used, such contexts might be a course, or a case, etc. It links content to more relevant information that might benefit the learner.

4. Technical information:

HEAL metadata schema extends IMS specifications that includes metadata specification and a content packaging specification based on IEEE LTSC LOM as its base (IMS Global Learning Consortium, 2006). HEAL added specific elements required for healthcare education such as clinical history and disease process (Dennis et al., 2004). It consists of over seventy elements and sub-elements organized in 9 categories (figure 5). Most of the elements are optional and the mandatory fields for each category are minimal. HEAL is implemented in XML to ensure its interoperability and it uses a controlled 
vocabulary such as MeSH, SNOMED, and UMLS. Also, it uses controlled vocabulary for describing elements such as formats and target audience.

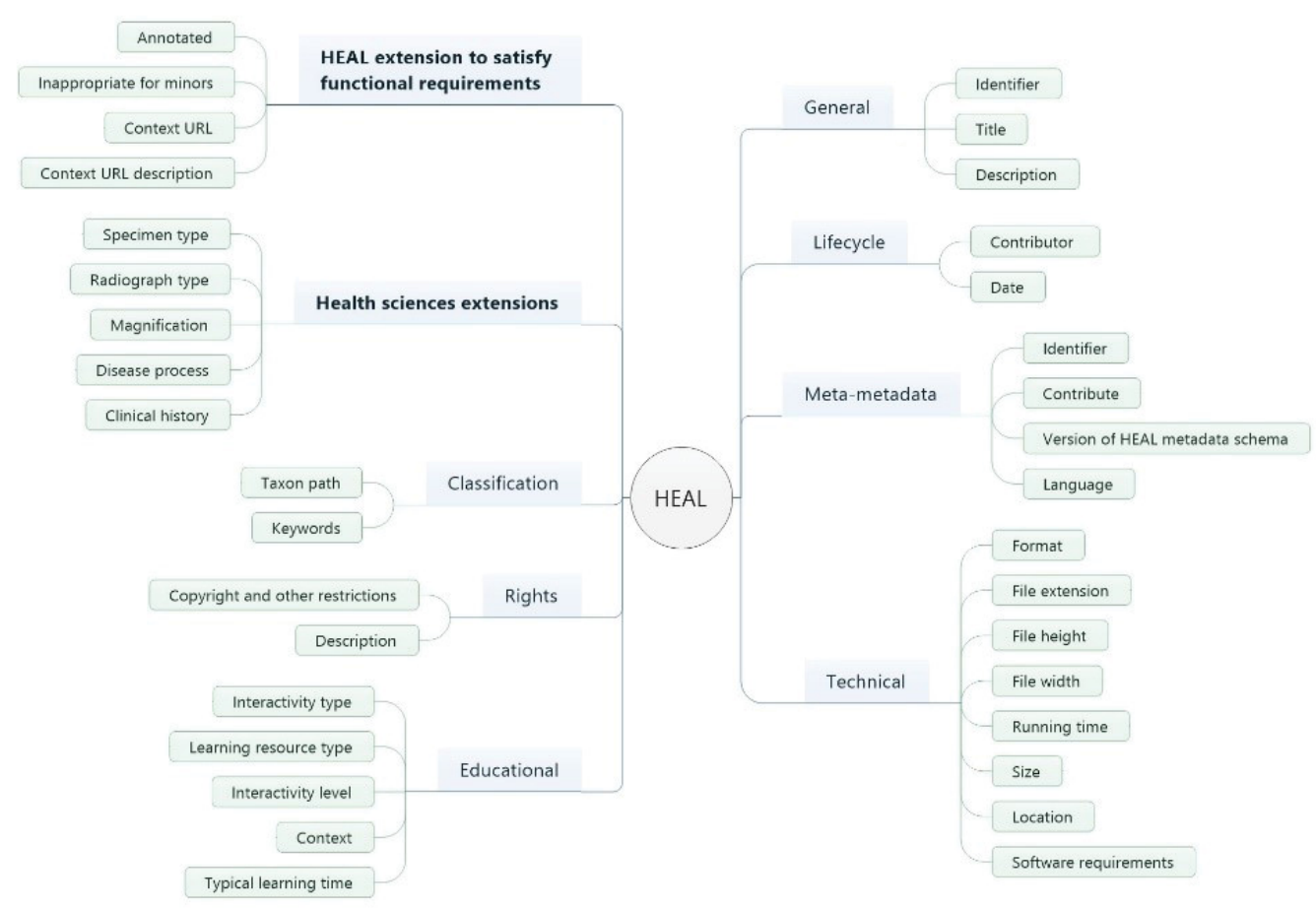

Figure 5: HEAL application profile

\subsection{NLM}

Figure National Library of Medicine, on the campus of the National Institute of Health in Bethesda, Maryland, USA, is the world's largest biomedical library (NLM, 2010). It maintains and makes a large volume of printed collections and datasets available on a wide range of topics to be searched by millions around the globe. NLM application profile (NLM, 2004) is based on the Dublin Core Metadata Initiative and incorporates additional elements identified as requirements by NLM for publishing its content. Results of evaluating the NLM against the evaluation matrix criteria are detailed as follows:

1. Educational objectives and fulfillment of learning needs:

As in the DCMI standard, NLM has the targeted audience element. It does not contain any elements for describing the educational needs or objectives of a resource. There exist a Notes element in the description category that is a free text space. It can be used to give additional information about the resource such as its educational needs or objectives, but this is not stated explicitly.

2. Description of Materials:

The NLM application profile provides a tile, a subject, table of content, and abstract elements to summarize the content of the resource described. Dates fields for capturing the date of creation, date of issue, date of the last update, major revision dates, and expiry date are also supported by the NLM application profile. Information about the digital appearance of the resource, such as software or hardware requirements needed, duration, or size of the resource can be stated. Another addition by NLM is an element that is responsible of supplying the name of a specific act or regulation if the creation or providing of the described resource requires specific legal instruments.

3. Provider details:

In addition to the publisher field, NLM application profile provides Contributor Name and Contributor Role elements to record information about the providers of the resource and their organizations. The NLM application profile added elements to state the extent to which a user can be assured that the resource will remain stable and available and records the agency responsible for guaranteeing the stability of that resource.

4. Technical information: 
The schema is based on the DCMI standard which is implemented in both XML and RDF/XML. Controlled Vocabularies are also used in this schema in multiple fields such as MeSH vocabulary that is used for describing the subject of the resource. IPR rights are explicitly declared in a specific field to guarantee the rights of usage for a resource.

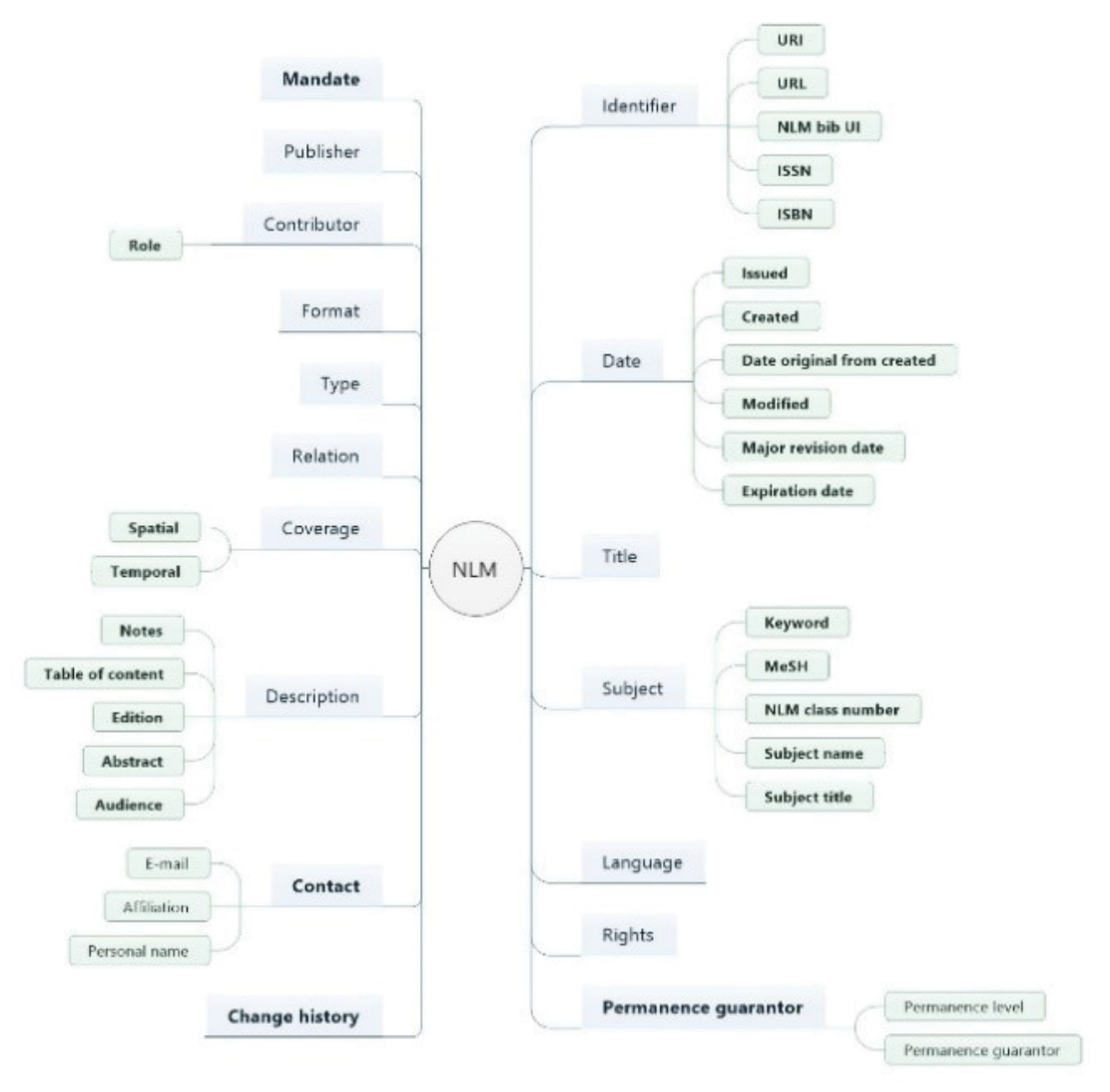

Figure 6: NLM application profiel

\section{Discussion}

The results highlight the general metadata practices used when implementing a metadata model for the medicine and healthcare e-learning materials and their alignment to the guidelines set for accreditation published by the EACCME. The study showed that the models meet part of the accreditation standards concerning the description of the materials, if publishing companies filled all the metadata properties in any of these models when used. Therefore, complying to the application profiles requirements for the metadata models when used, allow e-learning resources published in digital libraries to be mapped to the accreditation standards of EACCME. This section discusses the results of the comparison study and highlights the limitations found in the sample studied. The comparison results are detailed in table 2 . It has led to the following observations regarding the alignment of the application profiles against the selected criteria explained in the methodology.

- Educational objectives and fulfillment of learning needs: as stated in the EACCME, all the application profiles have an element to record the targeted audience, whether it is specified using age range or the educational level it is targeting. However, elements used to explicitly state the educational needs and/or objectives of the resources were applied only by mEducator application profiles. The other 3 , have elements such as description, or summary of the material that can be used to include the educational needs and objectives of the resource being described.

- Description of Material: all the application profiles, in the study, have a summary element to provide a summary for the resource being described, and elements to record important dates related to the resource such as creation date, published date, modification date, and expiry date. Nonetheless, a different set of dates elements are applied in each application profile. For example, the expiry date is stated in HealthCare LOM and NLM, but not applied in the others. The format required for use of the 
material is explicitly stated in all the application profiles. However, the duration needed to complete the described material is stated in two profiles only. As for the ethical and legal requirements, HEAL and NLM APs have elements to state that the resource complies with any ethical or legal needs. However, none of the application profiles have an explicit element to state that they respect learners' privacy and confidentiality. That is due to the diverse types of e-learning material they describe which is not all of them are of an interactive nature.

- Details of provider: At least the name of the individuals or the organizations involved in preparing the resources described are stated in all the application profiles. NLM provides additional contact that is responsible for maintaining the stability of the resource. Only HealthCare LOM clearly provide an element responsible for recoding the financial funding provided creating an e-learning material. On the other hand, HEAL provides hot links for information related to the material content or its providers.

- Technical implementation: All the application profiles were extended from the existing well-established metadata standards that were covered in the background section of this study. The implementation technology applied when developing the application profiles are XML and RDF, and different types of controlled vocabularies have been used to control the content for some elements such as subject or classification. mEducator and NLM use creative commons as IPR licensing for e-learning materials published. This guarantees the rights of providers and state clear usage declaration.

It is noticeable from the evaluation matrix in table 2 that the application profiles studied focused on using new technologies for enhancing the description and publishing of their content. This is due to the need for better discoverability and enhancing the searchability of online libraries using these application profiles. Other important properties such the source of funding for preparing the material for example, was stated clearly in only one application profile. On the other hand, the date of preparation of the material, and any revisions was provided in the properties of all the metadata model studied, and this is considered as very important factor in the accreditation standards of medical educational content. This evaluation matrix shows that some application profiles, such as mEducator for example, covered all the criteria in one category but failed to do so in another. All the application profiles failed to cover all the criteria of the EACCME explicitly in standalone properties in their application profiles.

Insufficient documentation of the mapping process for the application profiles was one of the main limitations in this study. For example, mEducator AP (figure 4) extends the DCMI standard (figure 2) but the exact additions and alternation of elements for the original DCMI standard are not clear in the documentation (Hendrix et al., 2012). The study highlighted the need for specific properties used for describing $r$ medicine and health care educational material such as the clinical history and disease process elements introduced the HEAL AP. The richness of the medical field with biomedical ontologies and controlled vocabulary, allowed such advancements to happen in the e-learning materials management on the web. Such ontologies and controlled vocabularies are used for managing and integrating of medical data in general. In education, the controlled vocabularies such as MeSH were highly integrated in most of the application profiles to describe the e-learning materials using subject headings that classify and ease the searchability of the materials (Fok and Ip, 2007). Most of the application profiles studied in this paper were implemented in some digital libraries and online repositories for hosting educational content in medicine and healthcare either by using all the APs properties, or part of their properties that is considered important for these hosting organizations. 
Table 2: The evaluation matrix and results of the comparison

\begin{tabular}{|c|c|c|c|c|}
\hline Analysis Criteria / Application profiles & HealthCareLOM & mEducator & HEAL & NLM \\
\hline \multicolumn{5}{|l|}{ Educational objectives and fulfillment of learning needs } \\
\hline Educational needs & No & Yes & No & No \\
\hline Expected educational outcomes & No & Yes & No & No \\
\hline Targeted Audience & Yes & Yes & Yes & Yes \\
\hline \multicolumn{5}{|l|}{ Description of Material } \\
\hline Brief summary & Yes & Yes & Yes & Yes \\
\hline $\begin{array}{l}\text { Respect and confirm learner privacy and } \\
\text { confidentiality }\end{array}$ & No & No & No & No \\
\hline $\begin{array}{l}\text { Duration needed by the learner to fulfill the } \\
\text { educational objectives of this material }\end{array}$ & Yes & No & Yes & Yes \\
\hline $\begin{array}{l}\text { Compliance of the material with all relevant ethical } \\
\text { and legal requirements }\end{array}$ & No & No & Yes & Yes \\
\hline $\begin{array}{l}\text { Date of preparation of the material, and any revisions } \\
\text { to its content and expiry date }\end{array}$ & Yes & Yes & Yes & Yes \\
\hline The required format for use of the material & Yes & Yes & Yes & Yes \\
\hline \multicolumn{5}{|l|}{ Details of the Provider } \\
\hline Description of the provider organization & Yes & Yes & Yes & Yes \\
\hline $\begin{array}{l}\text { Names and qualifications of individuals involved in } \\
\text { preparing the content }\end{array}$ & Yes & Yes & Yes & Yes \\
\hline $\begin{array}{l}\text { Name and title of the medical practitioner who will } \\
\text { take responsibility for its content }\end{array}$ & No & No & No & Yes \\
\hline $\begin{array}{l}\text { Declaration of actual or potential conflict of interest } \\
\text { of the individuals involved in preparing the material }\end{array}$ & No & No & No & No \\
\hline $\begin{array}{l}\text { Declare the source of funding provided for the } \\
\text { preparation of the material if applicable }\end{array}$ & Yes & No & No & No \\
\hline Provide hot links for further relevant information & No & No & Yes & No \\
\hline \multicolumn{5}{|l|}{ Technical Details } \\
\hline Base Schema & IEEE LOM & DCMI & IMS & DCMI \\
\hline XML binding technology & Yes & Yes & Yes & Yes \\
\hline RDF binding technology & Yes & Yes & No & Yes \\
\hline Used of controlled vocabulary & Yes & Yes & Yes & Yes \\
\hline IPR electronic licensing & No & Yes & No & Yes \\
\hline
\end{tabular}

\section{Conclusion}

This paper conducted a comparative study between application profiles of metadata models that were developed by organizations specialized in medicine and healthcare to manage publishing e-learning materials developed that field. This comparison study was conducted based on the accreditation criteria for the EACCME. Additional technical criteria include using specialized ontologies and publishing techniques were added to the comparison. The alignment of e-learning materials description with the EACCME standards guarantees having the best practices for describing and educational medical material. The application profiles studied had features that enhanced the technical requirements for describing its content than the qualitative properties that is important for being accredited by an organization such as EACCME. This study showed that designing application profiles and metadata models for describing educational medical content was based on the expertise of the organization developing such models without referring to international standards used for accrediting online content. This work, if combined with a study of users (learners and educators) behaviors in searching for educational materials, can provide valuable input towards best practices for metadata model applied on medicine and health care educational materials. One of the limitations for this study is that the comparative 
analysis of some entries was based on the documentation available in publications or the websites provided which is, in several cases, not enough to gather enough information about its implementation. Furthermore, the study should continue to analyze how such application profiles are applied by online repositories and digital repositories, and study if all the elements are used to fill the metadata of the ELMs. Since application profiles are highly aligned with the accreditation standards, then, if applied correctly, online repositories can easily get accredited if the quality of content is high.

\section{Refeerences}

Ashraf, J., Hussain, O. K. and Hussain, F. K., 2014. Making sense from Big RDF Data: OUSAF for measuring ontology usage, Software: Practice and Experience, p. n/a-n/a. doi: 10.1002/spe.2272.

Barker, P. and Campbell, L. M., 2010. metadata for learning materials: an overview of existing standards and current developments, Technology, Instruction, Cognition and Learning, 7(4), pp. 225-243 [online] Available at: http://www.icbl.hw.ac.uk/publicationFiles/2010/TICLMetadata/TICLpaper.MetadataForEducation_postref.pdf. [Accessed 25 August 2020]

Bodenreider, O., 2004. The Unified Medical Language System (UMLS): integrating biomedical terminology, Nucleic Acids Research. Narnia, 32(90001), p. 267D-270. doi: 10.1093/nar/gkh061.

Candler, C., Uijtdehaage, S. and Dennis, S., 2003. Introducing HEAL: The Health Education Assets Library, Academic Medicine, 78(3), pp. 249-254. [online] Available at:

https://www.researchgate.net/publication/10859100_Introducing_HEAL_the_Health_Education_Assets_Library [Accessed 25 August 2020]

Caswell, T., Henson, S., Jensen, M. and Wiley, D. (2008) 'Open Content and Open Educational Resources: Enabling universal education', The International Review of Research in Open and Distributed Learning, 9(1). doi: 10.19173/irrodl.v9i1.469.

Creative Commons, 2017. About the Licenses - Creative Commons. [online] Available at: https://creativecommons.org/licenses/ [Accessed 25 August 2020]

Delf, P., 2013. Designing effective eLearning for healthcare professionals, Radiography. 19(4), pp. 315-320. doi: 10.1016/J.RADI.2013.06.002.

Dennis, S. E., Dippie, S. R., Candler, C. S., McIntyre, S. A. and Uijtdehaage, S. (2004) An indexing standard for sharing health education multimedia resources: the health education assets library (HEAL) metadata schema, in Proceedings of the 37th Annual Hawaii International Conference on System Sciences (HICSS'04). Big Island, Hawaii: IEEE, pp. 138-147. doi: 10.1109/HICSS.2004.1265358.

Dhammi, I. K. and Kumar, S., 2014,.Medical Subject Headings (MeSH) terms, Indian Journal of Orthopaedics, 48(5), pp. 4434. doi: 10.4103/0019-5413.139827.

European Accreditation Council for CME (EACCME), 2017. Main UEMS [online] EACCME. Available at: https://www.uems.eu/areas-of-expertise/cme-cpd/eaccme [Accessed 25 August 2020]

Fleiszer, D. M., Posel, N. H. and Steacy, S. P., 2004. New directions in medical e-Curricula and the use of digital repositories, Academic Medicine, 79(3), pp. 229-235. doi: 10.1097/00001888-200403000-00006.

Fok, A. W. P. and Ip, H. H. S., 2007. Educational ontologies construction for personalized learning on the web, In: L. C., Jain, R. A., Tedman, and D. K Tedman, (eds) Evolution of teaching and learning paradigms in intelligent environment. studies in computational Intelligence. 62nd ed, Berlin, Heidelberg: Springer, pp. 47-82. doi: 10.1007/978-3-54071974-8_4.

Graves, M., Constabaris, A. and Brickley, D., 2007. FOAF: connecting people on the semantic web, Cataloging \& Classification Quarterly, 43(3-4), pp. 191-202.

Hendrix, M., Protopsaltis, A., Dunwell, I., de Freitas, S., Petridis, P., Arnab, S., Dovrolis, N., Kaldoudi, E., Taibi, D., Mitsopoulou, E., Dietze, S., Spachos, D. and Bamidis, P. (2012) 'Technical Evaluation of The mEducator 3 . 0 Linked Data- based Environment for Sharing Medical Educational Resources', in 2nd International Workshop on Learning and Education with the Web of Data. Lyon, France.

Hersh, W. R., Bhupatiraju, R. T., Greene, P., Smothers, V. and Cohen, C. (2006) 'Adopting e-learning standards in health care: competency-based learning in the medical informatics domain.', AMIA. Annual Symposium proceedings. AMIA Symposium. American Medical Informatics Association, 2006, pp. 334-338. Available at: http://www.ncbi.nlm.nih.gov/pubmed/17238358 [Accessed: 25 May 2019].

Hitzler, P. and Janowicz, K., 2013. Linked data, big data, and the 4th paradigm, Semantic Web, 4, pp. 233-235. doi: $10.3233 / \mathrm{SW}-130117$.

IEEE Computer Society, 2002. 1484.12.1-2002 - IEEE Standard for Learning Object Metadata. [online] IEEE. Available at: https://ieeexplore.ieee.org/document/1032843 [Accessed 25 August 2020]

IMS Global Learning Consortium, 2006, IMS meta-data best practice guide for IEEE 1484.12.1-2002 Standard for Learning Object Metadata. [online]I MS Global Consortium. Available at:

https://www.imsglobal.org/metadata/mdv1p3/imsmd_bestv1p3.html\#1621637 [Accessed: 3 July 2019].

International Standards Organisation, 2017. ISO 15836-1:2017 - Information and documentation -- The Dublin Core metadata element set -- Part 1: Core elements. [online] Available at: https://www.iso.org/standard/71339.html [Accessed: 30 June 2019]. 
International Standards Organisation,, 2019,.Standards in our world, consumers and standards: partnership for a better world. [online] Available at: https://www.iso.org/sites/ConsumersStandards/1_standards.html [Accessed: 24 May 2019].

Issam, R., Passardiere, D. E. L. A. and Jean-Marc, L., 2008. A formalism to describe open standards in order to generate application profiles, In: The 16th International Conference on Computer in Education. Taiwan, pp. 491-498.

Konopka, B. M., 2015. Biomedical ontologies - a review, Biocybernetics and Biomedical Engineering. pp. 75-86. doi: 10.1016/j.bbe.2014.06.002.

Konstantinidis, S., Kaldoudi, E. and Bamidis, P., 2009. Enabling content sharing in contemporary medical education: a review of technical standards, The Journal on Information Technology in Healthcare, 7(6), pp. 363-375. [online] Available at: http://citeseerx.ist.psu.edu/viewdoc/download?doi=10.1.1.477.4597\&rep=rep1\&type=pdf. [Accessed 25 August 2020]

Koutsomitropoulos, D. A., Alexopoulos, A. D., Solomou, G. D. and Papatheodorou, T. S. (2010) 'The Use of Metadata for Educational Resources in Digital Repositories', D-Lib Magazine, 16(1/2), pp. 1-15. doi: 10.1045/january2010koutsomitropoulos.

Maicher, L., 2007. Mapping between the Dublin Core Abstract Model DCAM and the TMDM, In: L. Maicher. L.M. Garshol (eds) Scaling topic maps. TMRA 2007. Lecture Notes in Computer Science, vol4999. Springer, Berlin, Heidelberg, pp. 198-213. doi: 10.1007/978-3-540-70874-2_19.

Miles, A., Matthews, B., Wilson, M. and Brickley, D. (2005) 'SKOS Core: Simple knowledge organisation for the Web | Miles | International Conference on Dublin Core and Metadata Applications', in DC-2005-Madrid Proceedings. Available at: https://dcpapers.dublincore.org/pubs/article/view/798 [Accessed: 27 April 2020].

National Library of Medicine, 2004. NLM Metadata Schema. U.S. National Library of Medicine. [online] Available at: https://www.nlm.nih.gov/tsd/cataloging/metafilenew.html [Accessed 3 July 2019].

National Library of Medicine, 2010. About the National Library of Medicine. U.S. National Library of Medicine. [online] Available at: https://www.nlm.nih.gov/about/index.html [Accessed: 3 July 2019].

Powell, A., Nilsson, M., Naeve, A. and Johnston, P. (2007) DCMI Abstract Model, Dublin core metadata initiative. Available at: http://dublincore.org/documents/2005/03/07/abstract-model/ [Accessed: 29 June 2019].

Reid, H. J., Thomson, C. and McGlade, K. J., 2016. Content and discontent: a qualitative exploration of obstacles to elearning engagement in medical students, BMC Medical Education., 16(1), p. 188. doi: 10.1186/s12909-016-0710-5.

Smothers, V., 2015. Healthcare learning object metadata specifications and description document. [online] Available at: https://www.medbiq.org/sites/default/files/files/HealthcareLOMSpecifications_pointrelease.pdf [Accessed 25 August 2020]

Stearns, M. Q., Price, C., Spackman, K. A. and Wang, A. Y. (2001) SNOMED clinical terms: overview of the development process and project status., Proceedings. AMIA Symposium. American Medical Informatics Association. Available at: http://www.ncbi.nlm.nih.gov/pubmed/11825268 [Accessed: 1 July 2019].

UEMS, 2016a. EACCME criteria for the accreditation of eLearning materials (ELM). [online] Available at: https://eaccme.uems.eu/img/user/files/UEMS2016_21 - EACCME 2_0 CRITERIA FOR THE ACCREDITATION OF ELM.pdf [Accessed 25 August 2020]

UEMS, 2016b. EACCME criteria for the accreditation of live educational events (LEE). [online] Available at: https://www.uems.eu/_data/assets/pdf_file/0016/40156/EACCME-2-0-CRITERIA-FOR-THE-ACCREDITATION-OF-LEEVersion-6-07-09-16.pdf [Accessed 25 August 2020]

W3C, 2014. RDF - Semantic web standards.[online] Available at: https://www.w3.org/RDF/ [Accessed 25 August 2020] 